\title{
CLASS A OPERATORS AND THEIR EXTENSIONS
}

\author{
SUNGEUN JUNG AND EUNGIL Ko
}

Abstract. In this paper, we study various properties of analytic extensions of class $A$ operators. In particular, we show that every analytic extension of a class $A$ operator has a scalar extension. As a corollary, we get that such an operator with rich spectrum has a nontrivial invariant subspace.

Mathematics subject classification (2010): Primary 47A11, Secondary 47A15, 47B20.

Keywords and phrases: Class A operators, subscalar operators, invariant subspaces, Weyl's theorem.

\section{REFERENCES}

[1] A. Aluthge, On p-hyponormal operators for $0<p<1$, Int. Eq. Op. Th. 13 (1990), 307-315.

[2] A. Aluthge And D. WAng, w-Hyponormal operators, Int. Eq. Op. Th. 36 (2000), 1-10.

[3] A. Aluthge And D. WAng, w-Hyponormal operators II, Int. Eq. Op. Th. 37 (2000), 324-331.

[4] S. BRown, Hyponormal operators with thick spectrum have invariant subspaces, Ann. of Math. 125 (1987), 93-103.

[5] X. CAO, Analytically class A operators and Weyl's theorem, J. Math. Anal. Appl. 320 (2006), 795803.

[6] I. COLOJOARǍ AND C. FoiAş, Theory of generalized spectral operators, Gordon and Breach, New York, 1968.

[7] J. B. Conway, A course in functional analysis, Springer-Verlag New York, 1990.

[8] J. EsCHMEIER, Invariant subspaces for subscalar operators, Arch. Math. 52 (1989), 562-570.

[9] C. FoIAS AND A. E. FraZHO, The commutant lifting approach to interpolation problem, Operator Theory Adv. Appl., vol. 44, Birkhä user, Boston, 1990.

[10] T. FURUTA, Invitation to linear operators, Taylor and Francis, 2001.

[11] J. K. HAN, H. Y. LEE, AND W. Y. LEE, Invertible completions of $2 \times 2$ upper triangular operator matrices, Proc. Amer. Math. Soc. 128 (1999), 119-123.

[12] M. ITO AND T. YAmAZAKI, Relations between two inequalities $\left(B^{\frac{r}{2}} A^{p} B^{\frac{r}{2}}\right)^{\frac{p}{p+r}} \geqslant B^{r}$ and $A^{p} \geqslant$ $\left(A^{\frac{p}{2}} B^{r} A^{\frac{p}{2}}\right)^{\frac{p}{p+r}}$ and their applications, Int. Eq. Op. Th. 44 (2002), 442-450.

[13] I. H. JeON AND B. P. DugGal, On operators with an absolute value conditions, J. Korean Math. Soc. 41 (2004), 617-627.

[14] S. Jung, E. Ko, And M. LeE, On class A operators, Studia Math. 198 (2010), 249-260.

[15] I. B. Jung, E. Ko, AND C. PEARCY, Aluthge transforms of operators, Int. Eq. Op. Th. 37 (2000), 449-456.

[16] I. H. KIM, On ( $p, k)$-quasihyponormal operators, Math. Ineq. Appl. 7 (2004), 629-638.

[17] E. Ko, K th roots of p-hyponormal operators are subscalar operators of order $4 k$, Int. Eq. Op. Th. 59 (2007), 173-187.

[18] K. Laursen And M. Neumann, An introduction to local spectral theory, Clarendon Press, Oxford, 2000.

[19] W. Y. LEE, Weyl's theorem for operator matrices, Int. Eq. Op. Th. 32 (1998), 319-331.

[20] W. Y. LEE, Weyl spectra of operator matrices, Proc. Amer. Math. Soc. 129 (2000), 131-138.

[21] W.Y. LEE AND S. H. LEE, A spectral mapping theorem for the Weyl spectrum, Glasgow Math. J. 38 (1996), 61-64. 
[22] M. OudghiRI, Weyl's theorem and perturbations, Int. Eq. Op. Th. 53 (2005), 535-545.

[23] S. M. Patel, M. Cho, K. TAnahas hi, And A. UChiyama, Putnam's inequality for class A operators and an operator transform by Cho and Yamazaki, Scientiae Mathematicae Japonicae 67 (2008), 393-402.

[24] C. M. PeARCY, Some recent developments in operator theory, Conference board of the mathematical sciences regional conference series in mathematics, 36 (1975).

[25] M. PUTINAR, Hyponormal operators are subscalar operators, J. Op. Th. 12 (1984), 385-395.

[26] M. PutinaR, Quasisimilarity of tuples with Bishop's property ( $\beta$ ), Int. Eq. Op. Th. 15 (1992), 1047 1052.

[27] K. Tanahashi, A. Uchiyama, AND M. Cho, Isolated points of spectrum of $(p, k)$ quasihyponormal operators, Linear Alge. Appl. 382 (2004), 221-229.

[28] D. Wang And J. I. LeE, Spectral properties of class A operators, Trends in Math, Information Center for Math. Science, 6 (2003), 93-98. 